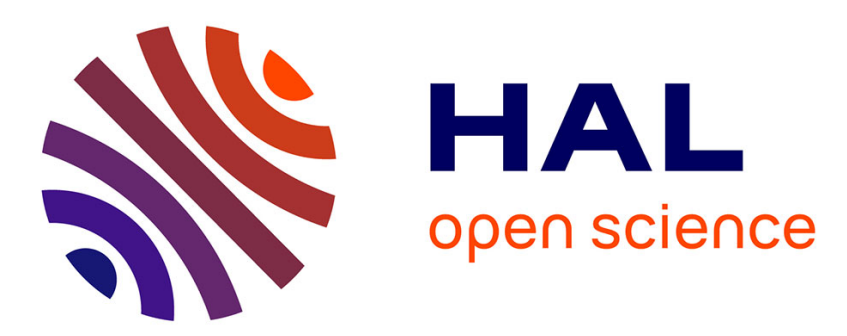

\title{
State estimation of system with bounded uncertain parameters: interval multi-model approach
}

Dalil Ichalal, Benoît Marx, Didier Maquin, José Ragot

\section{To cite this version:}

Dalil Ichalal, Benoît Marx, Didier Maquin, José Ragot. State estimation of system with bounded uncertain parameters: interval multi-model approach. International Journal of Adaptive Control and Signal Processing, 2018, 32 (3), pp.480-493. 10.1002/acs.2855 . hal-01657658

\section{HAL Id: hal-01657658 https://hal.science/hal-01657658}

Submitted on 17 Apr 2019

HAL is a multi-disciplinary open access archive for the deposit and dissemination of scientific research documents, whether they are published or not. The documents may come from teaching and research institutions in France or abroad, or from public or private research centers.
L'archive ouverte pluridisciplinaire HAL, est destinée au dépôt et à la diffusion de documents scientifiques de niveau recherche, publiés ou non, émanant des établissements d'enseignement et de recherche français ou étrangers, des laboratoires publics ou privés. 


\title{
State estimation of system with bounded uncertain parameters: interval multi-model approach
}

\author{
Dalil Ichalal $^{\mathrm{a}}$, Benoît Marx ${ }^{\mathrm{b}, \mathrm{c}}$, Didier Maquin ${ }^{\mathrm{b}, \mathrm{c}}$, José Ragot ${ }^{\mathrm{b}, \mathrm{c}}$ \\ ${ }^{a}$ IBISC-Lab, Evry Val d'Essonne University, 40, rue de Pelvoux, 91020, Evry \\ Courcouronnes, France. \\ ${ }^{b}$ Université de Lorraine, CRAN, UMR 7039, 2 avenue de la Forêt de Haye, 54516 \\ Vandoeuvre-lès-Nancy, France. \\ ${ }^{c} C N R S, C R A N$, UMR 7039, France
}

\begin{abstract}
The objective of this study is the analysis of dynamic systems represented by multi-model with variable parameters. Changes in these parameters are unknown but bounded. Since it is not possible to estimate these parameters over time, the simulation of such systems requires to consider all possible values taken by these parameters. More precisely, the goal is to determine, at any moment, the smallest set containing all the possible values of the state vector simultaneously compatible with the state equations and with a priori known bounds of the uncertain parameters. This set will be characterized by two trajectories corresponding to the lower and upper limits of the state at every moment. This characterization can be realized by a direct simulation of the system, given the bounds of its parameters. It can also be implemented with a Luenberger type observer, fed with the system measurements.
\end{abstract}

Keywords: Interval Observers, Uncertain Systems, Bounded Uncertainties, Multi-Model Technique

\section{Introduction}

As widely known, one of the main difficulties in system simulation, control or estimation is to deal with uncertainties. These uncertainties may affect the input or output signals of the system (e.g. unknown input, disturbance, measurement noises, etc) as well as the system model itself (e.g. non modeled dynamics, unknown parameter, etc). The uncertainties may also be of different natures: total lack of information (unknown value of a parameter) or partial knowledge (upper and lower bounds, statistical properties, etc). The proposed paper deals with system with uncertain parameters.

Email addresses: dalil.ichalal@ibisc.univ-evry.fr (Dalil Ichalal), benoit.marx@univ-lorraine.fr (Benoît Marx), didier.maquin@univ-lorraine.fr (Didier Maquin), jose.ragot@univ-lorraine.fr (José Ragot) 
Even a low magnitude change in some parameters may have a significant impact on the system behavior, and namely on the system state trajectory. Uncertain parameters can be considered from two main points of view: the stochastic and the deterministic ones. In the first approach, the uncertain parameters are assumed to be the results of random process realizations. It then needs to chose the probability density functions and their parameters describing the system uncertain parameters. In the deterministic approach, no statistical models of the parameters are assumed to be available and only upper and lower bounds of the parameter values are known. This approach, also known as the interval approach, is adopted in the present paper.

The search for trajectories which are solutions of differential inclusions [32] were the starting point of a large amount of works on stability, stabilization and state estimation. In the latter topic our communication follows the pioneering work of Gouzé [21] that proposed the synthesis of interval type observer to reconstruct the system state from measurements of inputs and outputs. Since then, many results were published on designing observers adapted to nonlinear systems with uncertain but bounded parameters [11], [12], [6], [9].

The model-based state estimation techniques in the context of bounded uncertainties can be classified into two categories. The first one is based on the mechanism "prediction/correction" [24], [33]. Specifically, based on information at time $k$, the dynamic state equation provides the possible state set at the time $k+1$ through a one step prediction. At this $k+1$ instant, the available measures can also be used to provide a state set by inverting the output equations. The correction phase, which proceeds by searching for the intersection of the two sets obtained above, then provides the estimated state compatible with the model, its uncertainties and the measures. The second approach [7], [15], [4], [40], [36] consists in explicitly building an interval observer from the interval model of the system. Then, solving the interval state equations of the observer, upper and lower bounds of the state variable estimates are provided.

Studies on systems with bounded uncertain parameters can be found in several areas of automatic control: stability analysis [30], [18], state estimation [16] [14] [33], synthesis of iterative learning control law [1], system diagnosis [27] [22], components fault detection [25], [34], data analysis [23], model sensitivity analysis [35], regression with abnormal values [39], classification [38]. Roughly speaking, uncertainties in the measures and in the parameters are handled in two main ways. In the first one, under robustness constraints, aims to develop analysis and synthesis techniques insensitive or partially immune to uncertainties. This leads in most cases to minimize a criterion reflecting the impact of these uncertainties on the objective to be pursued in terms of control or state estimation. In the second one, the aim is to quantify the impact of the uncertainties in the objective to pursue. The objective can be, for instance, to quantify the influence of uncertain and bounded parameters on the estimated system state, leading to analyze the uncertainty propagation throughout the control and state estimation procedures.

Up to the authors' knowledge, the use of observer adapted to real processes with bounded uncertain parameter is barely exposed in the literature, never- 
theless, one may find some applications with only simulations or laboratory processes. In [26], an interval observer was synthesized to estimate the water quality in a distribution network. In [28] [31], [2] and [19] bio-processes were considered as applications for observer design, as well as in [6] with probabilistic uncertain parameters. In [8], a mechanical structure analysis with interval type parameters is proposed. In the field of transport, [20] and [40] presented the synthesis of an interval observer to estimate the vehicle position and velocity. The socio-economic field is no exception to the use of this approach, as shown for example in [39].

Our proposal also participates of this second approach, even if it differs markedly from two perspectives. First, based on bounded uncertainties, a multimodel approach provides two distinct models for the upper and lower bounds of the system state. Then, based on these two models, an interval Luenberger-like multi-observer is designed, to reconstruct the bounds of the system states from measurements of its inputs and outputs. The contributions of this paper is to develop an improved structure of an interval observer with an analytical form, to study its stability in order to compute its gains and to take into account the uncertainties affecting the system with the help of a polytopic representation.

The main required assumptions for the interval observer design to guarantee error boundedness is the exclusion of the zero value from the intervals defining the parameter uncertainties. Put in other words, it means that at least the signs of the uncertain parameters are known even if their exact values are not known. It can also be pointed out that our approach does not need to assume some structural requirements on the state matrix, such as being Metzler (off diagonal element should be positive) as often used in the literature [16], [6], [36], [40] (and references therein).

The paper is organized as follows. Section 2 is devoted to the interval formulation of a system with time varying bounded parameters. In section 3, the stability of the proposed structure is studied. This result is then exploited in section 4 to derive the observer synthesis. After some concluding remarks in section 5, an appendix details the derivation of the proposed multi-model approach.

\section{Interval model of a system with uncertain bounded parameters}

\subsection{Structure}

The discrete time representation (1) is considered, where $x \in \mathbb{R}^{n}$ is the state vector, $u \in \mathbb{R}^{m}$ the input, $y \in \mathbb{R}^{p}$ the measured output, $A_{k}$ and $B$ matrices of appropriate dimensions. Parametric variations $\Delta_{k}$ (which are not available for measurements) only affect the state matrix, taking into account changes in the $B$ and $C$ matrices being a simple extension.

$$
\left\{\begin{array}{l}
x_{k+1}=A_{k} x_{k}+B u_{k} \\
A_{k}=A+\Delta_{k} \\
y_{k}=C x_{k}
\end{array}\right.
$$


Hypothesis 1. Parametric variations $\Delta_{k}$ are bounded by

$$
-\Delta \leq \Delta_{k} \leq \Delta
$$

where the bound $\Delta$ is known and the inequalities in (2) are to be understood component-wise.

Hypothesis 2. The null matrix of the same size as those of $A$, does not belong to the interval matrix $[A-\Delta A+\Delta]$. In other words, the lower and upper bounds of each element of this interval matrix have the same sign.

From (1), one can build the state trajectories $\underline{x}_{k}$ and $\bar{x}_{k}$ containing the set of reachable states given the parametric variations $\Delta_{k}$. As it will be explained, the objective is to avoid to evaluate all the possible values of the uncertainties to determine all the possible corresponding state trajectories. What will be done is to recursively calculate only the upper and lower extremal trajectories of the state envelope and a proof will be provided to show that all the possible state trajectories are included in this envelope.

\subsection{Envelope of a state trajectory}

Proposition 1 (Product of two interval variables). Let us consider the scalar $p_{k}=\left(a+\delta_{k}\right) x_{k}$ where $\left|\delta_{k}\right| \leq \delta$ and where $x_{k}$ is an interval variable, namely $x_{k} \in\left[\underline{x}_{k} \bar{x}_{k}\right]$. To determine the bounds $\underline{p}_{k}$ and $\bar{p}_{k}$ of the interval variable $p_{k}$, two cases must be distinguished whether $a$ is positive or negative:

$$
\begin{gathered}
a>0\left\{\begin{aligned}
\underline{p}_{k} & =a \underline{x}_{k}-\delta\left|\underline{x}_{k}\right| \\
& =\underline{x}_{k}\left(a-\delta \operatorname{sgn}\left(\underline{x}_{k}\right)\right) \\
\bar{p}_{k} & =a \bar{x}_{k}+\delta\left|\bar{x}_{k}\right| \\
& =\bar{x}_{k}\left(a+\delta \operatorname{sgn}\left(\bar{x}_{k}\right)\right)
\end{aligned}\right. \\
a<0\left\{\begin{aligned}
\underline{p}_{k} & =a \bar{x}_{k}-\delta\left|\bar{x}_{k}\right| \\
& =\bar{x}_{k}\left(a-\delta \operatorname{sgn}\left(\bar{x}_{k}\right)\right) \\
\bar{p}_{k} & =a \underline{x}_{k}+\delta\left|\underline{x}_{k}\right| \\
& =\underline{x}_{k}\left(a+\delta \operatorname{sgn}\left(\underline{x}_{k}\right)\right)
\end{aligned}\right.
\end{gathered}
$$

Having considered the two cases (3) and (4), $p_{k}$ is expressed in respect to its two bounds as follows:

$$
\left\{\begin{aligned}
\underline{p}_{k}= & \frac{1}{2}\left[(a+|a|) \underline{x}_{k}+(a-|a|) \bar{x}_{k}\right] \\
& -\frac{1}{2}\left[\delta\left(\left|\underline{x}_{k}\right|+\left|\bar{x}_{k}\right|+\operatorname{sgn}(a)\left(\left|\underline{x}_{k}\right|-\left|\bar{x}_{k}\right|\right)\right)\right] \\
\bar{p}_{k}= & \frac{1}{2}\left[(a-|a|) \underline{x}_{k}+(a+|a|) \bar{x}_{k}\right] \\
& +\frac{1}{2}\left[\delta\left(\left|\underline{x}_{k}\right|+\left|\bar{x}_{k}\right|-\operatorname{sgn}(a)\left(\left|\underline{x}_{k}\right|-\left|\bar{x}_{k}\right|\right)\right)\right]
\end{aligned}\right.
$$


These results can directly be generalized to the case of matrix products as it appears in (1), which explicitly expresses the system state in an interval form, for any time $k$. The coupled recurrences are obtained and allow to recursively express the two bounds of the state [10]:

$$
\left\{\begin{aligned}
\underline{x}_{k+1}= & \frac{1}{2}\left[(A+|A|) \underline{x}_{k}+(A-|A|) \bar{x}_{k}\right]+B u_{k} \\
& -\frac{1}{2}\left[\left(\Delta+\Delta * S_{A}\right)\left|\underline{x}_{k}\right|-\left(\Delta-\Delta * S_{A}\right)\left|\bar{x}_{k}\right|\right] \\
\bar{x}_{k+1}= & \frac{1}{2}\left[(A-|A|) \underline{x}_{k}+(A+|A|) \bar{x}_{k}\right]+B u_{k} \\
& +\frac{1}{2}\left[\left(\Delta-\Delta * S_{A}\right)\left|\underline{x}_{k}\right|+\left(\Delta+\Delta * S_{A}\right)\left|\bar{x}_{k}\right|\right]
\end{aligned}\right.
$$

where $*$ symbolizes the Hadamard matrix product and $S_{A}=\operatorname{sgn}(A)$ (where the sign function is applied component-wise to the matrix $A$ ).

Proposition 2 (Interval trajectory of uncertain dynamic system). The system with bounded uncertain parameters (1) is characterized by an interval-type state which bounds are given by the following augmented system:

$$
\left[\begin{array}{l}
\underline{x}_{k+1} \\
\bar{x}_{k+1}
\end{array}\right]=M\left[\underline{x}_{k}\right]+N\left[\begin{array}{l}
\left|\underline{x}_{k}\right| \\
\bar{x}_{k}
\end{array}\right]+E B u_{k}
$$

with

$M=\frac{1}{2}\left[\begin{array}{ll}A+|A| & A-|A| \\ A-|A| & A+|A|\end{array}\right], \quad E=\left[\begin{array}{c}I_{n} \\ I_{n}\end{array}\right], \quad N=\frac{1}{2}\left[\begin{array}{rr}-\Delta-\Delta * S_{A} & -\Delta+\Delta * S_{A} \\ \Delta-\Delta * S_{A} & \Delta+\Delta * S_{A}\end{array}\right]$

Remark 1 (Computation of the $i^{t h}$ component of the interval state vector in the input free case $\left.u_{k}=0\right)$. Let us detail the computation of this component with respect to the lower bound of the system state (the calculation is similar to the upper bound). Denoting $a_{i, j}$ and $\delta_{i, j}$ the respective elements of matrices $A$ and $\Delta$, from (6) one gets:

$$
\begin{aligned}
\underline{x}_{k+1, i}= & \frac{1}{2} \sum_{j=1}^{n}\left(\left(a_{i, j}+\left|a_{i, j}\right|\right) \underline{x}_{k, j}+\left(a_{i, j}-\left|a_{i, j}\right|\right) \bar{x}_{k, j}\right) \\
& -\frac{1}{2} \sum_{j=1}^{n}\left(\delta_{i, j}\left(\left|\underline{x}_{k, j}\right|+\left|\bar{x}_{k, j}\right|\right)+\operatorname{sgn}\left(a_{i, j}\right) \delta_{i, j}\left(\left|\underline{x}_{k, j}\right|-\left|\bar{x}_{k, j}\right|\right)\right)
\end{aligned}
$$

Let us define the term $\sigma_{i, j}$ of rank $j$ in (9) such that $\underline{x}_{k+1, i}=\sum_{j=1}^{n} \sigma_{i, j}$. One can then simplify the expression of the term $\sigma_{i, j}$ with respect to the sign of the matrix A coefficients:

- If $a_{i, j}>0$

$$
\begin{aligned}
\sigma_{i, j} & =a_{i, j} \underline{x}_{k, j}-\frac{\delta_{i, j}}{2}\left(\left(\left|\underline{x}_{k, j}\right|+\left|\bar{x}_{k, j}\right|\right)+\left(\left|\underline{x}_{k, j}\right|-\left|\bar{x}_{k, j}\right|\right)\right) \\
& =a_{i, j} \underline{x}_{k, j}-\delta_{i, j}\left|\underline{x}_{k, j}\right|
\end{aligned}
$$


- If $a_{i, j}<0$

$$
\begin{aligned}
\sigma_{i, j} & =a_{i, j} \bar{x}_{k, j}-\frac{\delta_{i, j}}{2}\left(\left(\left|\underline{x}_{k, j}\right|+\left|\bar{x}_{k, j}\right|\right)-\left(\left|\underline{x}_{k, j}\right|-\left|\bar{x}_{k, j}\right|\right)\right) \\
& =a_{i, j} \bar{x}_{k, j}-\delta_{i, j}\left|\bar{x}_{k, j}\right|
\end{aligned}
$$

These results will be used later.

\subsection{Validation of the envelope of the state trajectory}

In (6) the two bounds $\underline{x}_{k}$ and $\bar{x}_{k}$ of the state were recursively defined and they were claimed to define an envelope of the original system with interval parameters (1). It still remains to prove that the envelope does contain the actual state of the system (1). For this, the following property is proven by induction on $k$ :

$$
\mathcal{H}_{k}: \underline{x}_{k} \leq x_{k} \leq \bar{x}_{k}
$$

By definition of $\underline{x}_{0}$ and $\bar{x}_{0}$ in (6), it holds that $x_{0} \in\left[\underline{x}_{0}, \bar{x}_{0}\right]$, so the property is satisfied for $k=0$. Assuming that for a given positive $k$ the property $\mathcal{H}_{k}$ is true, then let us show that $\underline{x}_{k+1} \leq x_{k+1}$ is true (the other inequality in (12) is proved similarly) implying that $\mathcal{H}_{k+1}$ is also true. Given (6) and (1), the distance between the actual state and the lower bound is deduced:

$$
\begin{aligned}
\underline{\tilde{x}}_{k+1}= & x_{k+1}-\underline{x}_{k+1} \\
= & \left(A+\Delta_{k}\right) x_{k}-\frac{1}{2}(A+|A|) \underline{x}_{k}-\frac{1}{2}(A-|A|) \bar{x}_{k} \\
& +\frac{1}{2}\left(\Delta+\Delta * S_{A}\right)\left|\underline{x}_{k}\right|-\frac{1}{2}\left(\Delta-\Delta * S_{A}\right)\left|\bar{x}_{k}\right|
\end{aligned}
$$

As reported in remark 1 , the analysis of this difference can be made for the $i^{\text {th }}$ component of $\tilde{x}_{k+1}$. To do this, substitute (9) and (1) in (13) to obtain:

$$
\begin{aligned}
\underline{\tilde{x}}_{k+1, i} & =x_{k+1, i}-\underline{x}_{k+1, i} \\
& =\sum_{j=1}^{n}\left(\left(a_{i, j}+\delta_{k, i, j}\right) x_{k, j}-\sigma_{i, j}\right)
\end{aligned}
$$

where $\delta_{k, i, j}$ denotes the $(i, j)$ entry of the matrix $\Delta_{k}$. In the sum appearing in (14), only the term $\tilde{\sigma}_{i, j}$ of rank $j$ is explained in view of the expression (10) and (10) of $\sigma_{i, j}$ :

- If $a_{i, j}>0$

$$
\begin{aligned}
\tilde{\sigma}_{i, j} & =\left(a_{i, j}+\delta_{k, i, j}\right) x_{k, j}-a_{i, j} \underline{x}_{k, j}+\delta_{i, j}\left|\underline{x}_{k, j}\right| \\
& \geq\left(a_{i, j}+\delta_{k, i, j} \underline{x}_{k, j}-a_{i, j} \underline{x}_{k, j}+\delta_{i, j}\left|\underline{x}_{k, j}\right|\right. \\
& \geq \delta_{k, i, j} \underline{x}_{k, j}+\delta_{i, j}\left|\underline{x}_{k, j}\right| \\
& \geq 0
\end{aligned}
$$

- If $a_{i, j}<0$

$$
\begin{aligned}
\tilde{\sigma}_{i, j} & =\left(a_{i, j}+\delta_{k, i, j}\right) x_{k, j}-a_{i, j} \bar{x}_{k, j}+\delta_{i, j}\left|\bar{x}_{k, j}\right| \\
& \geq\left(a_{i, j}+\delta_{k, i, j}\right) \bar{x}_{k, j}-a_{i, j} \bar{x}_{k, j}+\delta_{i, j}\left|\bar{x}_{k, j}\right| \\
& \geq \delta_{k, i, j} \bar{x}_{k, j}+\delta_{i, j}\left|\bar{x}_{k, j}\right|
\end{aligned}
$$


Finally, according to (14), (15) and (16), $\underline{\tilde{x}}_{k+1, i}$ is a sum of non negative terms and thus $x_{k+1, i} \geq \underline{x}_{k+1, i}$, i.e. $x_{k+1} \geq \underline{x}_{k+1}$. Thus, the property $\mathcal{H}_{k+1}$ has been demonstrated and the induction principle ensures that for positive $k$, $x_{k} \geq \underline{x}_{k}$. In the same way, by analyzing the quantity $\tilde{\bar{x}}_{k}=\bar{x}_{k}-x_{k}$, it can be established that $x_{k} \leq \bar{x}_{k}$. It can thus be concluded that (6) is an envelope of the system state trajectory.

\subsection{Example}

The considered example is a system (1) with $m=2$ inputs and $n=2$ state variables, defined by the matrices $A$ and $B$ given in (17). The parametric variations $\Delta_{k}$ affecting the state matrix take values between -0.01 and 0.01 and accordingly its bound (2) is defined by the matrix $\Delta$ in (17). Figure 1 displays the two inputs (top of the figure) and the two states (bottom of the figure). For each state variable, the red line is a possible state trajectory and the two others are the upper and lower bounds of the state trajectories according to the considered parameter uncertainties.

$$
A=\left[\begin{array}{cc}
0.925 & -0.05 \\
-0.25 & 0.25
\end{array}\right], \quad B=\left[\begin{array}{cc}
0.4 & 0 \\
0 & 0.5
\end{array}\right], \quad \Delta=\left[\begin{array}{cc}
0.01 & 0.01 \\
0.01 & 0.01
\end{array}\right]
$$
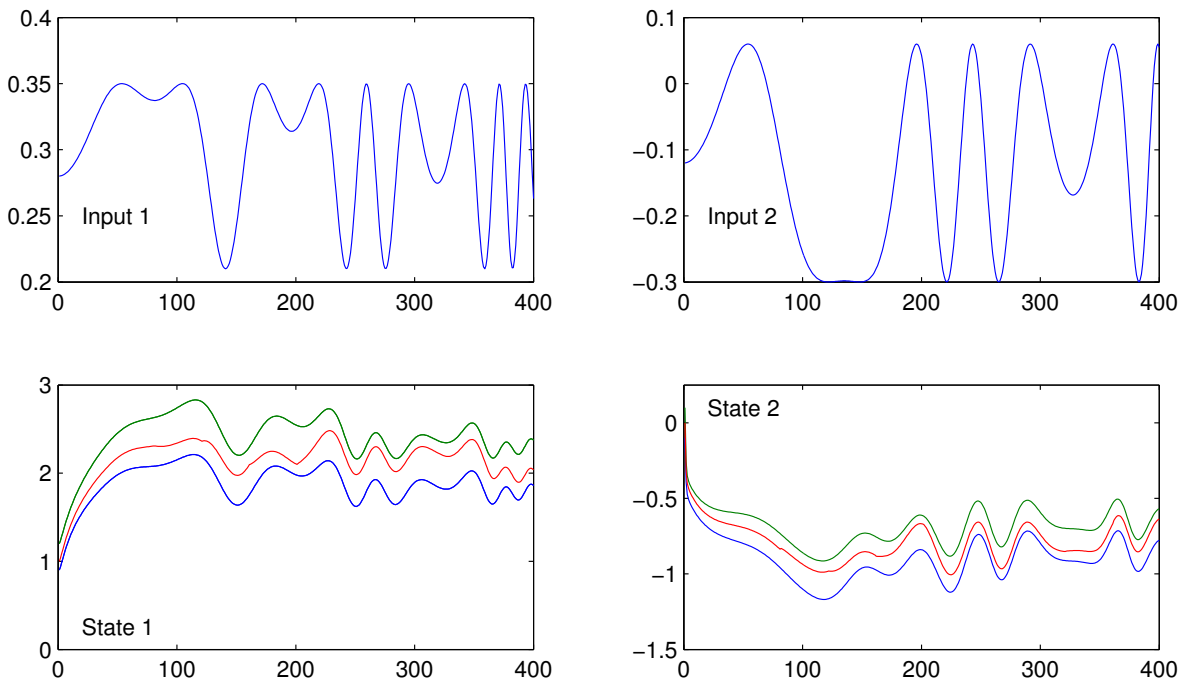

Figure 1: Simulation of a system with interval parameters

\section{Stability analysis}

In this section, the stability of interval systems with bounded uncertain parameters is studied with the second Lyapunov method, which is known to be devoted to the analysis of internal stability of dynamic systems. The principle of this method is to study the convergence of the system state $x$ to the origin. 
This is achieved through a scalar positive definite function of the state, denoted $V$, and called the Lyapunov candidate function.

Since no specific Lyapunov functions have already been proposed for interval models (6) defined by a generalized state encompassing the upper and lower bounds of the state as well as its absolute value, it is here proposed to transform the absolute value function using a nonlinear sector bounded approach. Section 3.1 is dedicated to this approach, which then allows the stability study in section 3.2 .

\subsection{New structure of the system model}

Defining the generalized state $z_{k}=\left[\begin{array}{ll}\underline{x}_{k}^{T} & \bar{x}_{k}^{T}\end{array}\right]^{T}$ the system (7) becomes:

$$
z_{k+1}=M z_{k}+N\left|z_{k}\right|+E B u_{k}
$$

with $M$ and $N$ defined in (8).

As mentioned in the preamble, the stability analysis is eased by writing the nonlinearity under a polytopic form depending on $\left|z_{k}\right|$.

For any scalar $\alpha$, the following decomposition holds:

$$
\left\{\begin{array}{l}
|\alpha|=\mu_{1}(\alpha) \cdot(\alpha)+\mu_{2}(\alpha) \cdot(-\alpha) \\
\mu_{1}(\alpha)=\frac{1+\operatorname{sgn}(\alpha)}{2} \\
\mu_{2}(\alpha)=1-\mu_{1}(\alpha)
\end{array}\right.
$$

which can also be written as:

$$
\left\{\begin{array}{l}
|\alpha|=\sum_{i=1}^{2} \mu_{i}(\alpha) E_{i} \alpha \\
E_{1}=1 \\
E_{2}=-1
\end{array}\right.
$$

As detailed in the appendix Appendix A, this writing directly extends to a vector $z \in \mathbb{R}^{2 n}$ (Appendix A):

$$
\left|z_{k}\right|=\sum_{j=1}^{r} \mu_{j}\left(z_{k}\right) E_{j} z_{k}, \quad r=2^{2 n}
$$

Finally, with (21), the interval model (18) is expressed under the polytopic form:

$$
z_{k+1}=\sum_{j=1}^{r} \mu_{j}\left(z_{k}\right)\left(M+N E_{j}\right) z_{k}+E B u_{k}
$$

where the activating functions $\mu_{j}$ satisfy the so-called convex sum property:

$$
\sum_{j=1}^{r} \mu_{j}\left(z_{k}\right)=1, \quad 0 \leq \mu_{j}\left(z_{k}\right) \leq 1, \forall k \in \mathbb{N}, \forall j=1, \ldots, r
$$




\subsection{Stability criteria}

Theorem 1. The asymptotical stability of the input free system (22) (i.e. with $\left.u_{k}=0\right)$ is guaranteed if there exists matrices $P=P^{T}>0$ and $G$ such that the LMI (24) holds.

$$
\left[\begin{array}{cc}
-P & \left(M+N E_{j}\right)^{T} G \\
G^{T}\left(M+N E_{j}\right) & P-G-G^{T}
\end{array}\right]<0, \quad j=1, \ldots, r
$$

Proof. Defining a quadratic Lyapunov function $V\left(z_{k}\right)=z_{k}^{T} P z_{k}$ with $P>0$, its variation along the state trajectory of (22), defined by $\Delta V_{k}=V\left(z_{k+1}\right)-V\left(z_{k}\right)$, is given by $\Delta V_{k}=z_{k}^{T} \mathcal{M} z_{k}$, with:

$$
\mathcal{M}=\left(M+N \sum_{j=1}^{r} \mu_{j}\left(z_{k}\right) E_{j}\right)^{T} P\left(M+N \sum_{j=1}^{r} \mu_{j}\left(z_{k}\right) E_{j}\right)-P
$$

Obviously, the negativity of the variation $\Delta V_{k}$ is implied if there exists $P=$ $P^{T}>0$ satisfying $\mathcal{M}<0$. From lemma 3 of [13], it is known that finding $P=P^{T}>0$ satisfying $\mathcal{M}<0$ is equivalent to find $P=P^{T}>0$ and $G$ satisfying

$$
\left[\begin{array}{cc}
-P & \left(M+N \sum_{j=1}^{r} \mu_{j}\left(z_{k}\right) E_{j}\right)^{T} G \\
G^{T}\left(M+N \sum_{j=1}^{r} \mu_{j}\left(z_{k}\right) E_{j}\right) & P-G-G^{T}
\end{array}\right]<0
$$

The positivity of the functions $\mu_{j}$ ensures that (24) are sufficient conditions to (26) and thus to $\Delta V_{k}<0$ and to the input free asymptotical stability of (22).

Corollary 1. The system (22) is asymptotically stable if there exists matrices $P_{i}=P_{i}^{T}>0$ and $G$ such that:

$$
\mathcal{M}_{i, j}+\mathcal{M}_{j, i}<0, i, j=1, \ldots, r
$$

where $\mathcal{M}_{i, j}$ is defined by:

$$
\mathcal{M}_{i, j}=\left[\begin{array}{cc}
-P_{i} & \left(M+N E_{i}\right)^{T} G \\
G^{T}\left(M+N E_{i}\right) & P_{j}-G-G^{T}
\end{array}\right]
$$

Proof. The proof is similar to the one of Theorem 1, with the Lyapunov candidate function defined by $V\left(z_{k}\right)=z_{k}^{T}\left(\sum_{i=1}^{r} \mu_{i}\left(z_{k}\right) P_{i}\right) z_{k}$ and the factorization of [13].

\section{Observer design for a system with bounded uncertain parameters}

\subsection{Observer structure}

Before detailing the proposed observer, let us recall that the state equation of the system (1) may also be described by its lower and upper bounds (6). 
Given the structure of (6), the proposed observer (29) is designed to provide an interval state estimate $\left[\begin{array}{ll}\underline{\hat{x}}_{k} & \hat{\bar{x}}_{k}\end{array}\right]$ which gives an envelope of the actual system state, based only on the input and output measurements of the system (1). Namely, it reduces to find the observer gains $L_{1}$ and $L_{2}$ such that $x_{k} \in\left[\underline{\underline{x}}_{k} \hat{\bar{x}}_{k}\right]$, for every instant $k$ and to impose some dynamics to the state reconstruction error.

$$
\left\{\begin{aligned}
\underline{\hat{x}}_{k+1}= & \frac{1}{2}\left[(A+|A|) \underline{\hat{x}}_{k}+(A-|A|) \hat{\bar{x}}_{k}\right]-\left(\Delta+\Delta * S_{A}\right)\left|\underline{\hat{x}}_{k}\right| \\
& \frac{-}{\left(\Delta-\Delta * S_{A}\right)\left|\hat{\bar{x}}_{k}\right|+L_{1}\left(y_{k}-C \hat{x}_{k}\right)+B u_{k}} \\
\hat{\bar{x}}_{k+1}= & \frac{1}{2}\left[(A-|A|) \underline{\hat{x}}_{k}+(A+|A|) \bar{x}_{k}\right]+\left(\Delta-\Delta * S_{A}\right)\left|\underline{\hat{x}}_{k}\right| \\
& +\left(\Delta+\Delta * S_{A}\right)\left|\hat{\bar{x}}_{k}\right|+L_{2}\left(y_{k}-C \hat{\bar{x}}_{k}\right)+B u_{k}
\end{aligned}\right.
$$

\subsection{Rationale for observer structure}

The rationale for this structure is done in two steps. First, it must be shown that $\underline{\hat{x}}_{k} \leq x_{k} \leq \hat{\bar{x}}_{k}$. Secondly, the observer gains $L_{1}$ and $L_{2}$ must be adjusted to specify the temporal characteristics of state reconstruction error. Each of these steps is addressed in Theorem 2 and 3 respectively.

Theorem 2. The system (29) is an observer for (1), i.e. the state of (1) belongs to the envelope defined by (29).

Proof. The proof proceeds by induction. Let $\mathcal{H}_{k}$ be the following property:

$$
\mathcal{H}_{k}: \underline{\hat{x}}_{k} \leq x_{k} \leq \hat{\bar{x}}_{k}
$$

The gaps between the state $x_{k}$ and the lower and upper bounds of its estimate are defined by:

$$
\underline{e}_{k}=x_{k}-\underline{\hat{x}}_{k} \quad \text { and } \quad \bar{e}_{k}=\hat{\bar{x}}_{k}-x_{k}
$$

The first one is expressed at time $k+1$, from (1) and (29), by:

$$
\begin{aligned}
\underline{e}_{k+1}= & \left(A+\Delta_{k}\right) x_{k}-\frac{1}{2}\left[(A+|A|) \underline{\hat{x}}_{k}+(A-|A|) \hat{\bar{x}}_{k}\right] \\
& -L_{1}\left(y_{k}-C \underline{\hat{x}}_{k}\right)-\frac{1}{2}\left[\left(\Delta+\Delta * S_{A}\right)\left|\underline{\hat{x}}_{k}\right|+\left(\Delta-\Delta * S_{A}\right)\left|\hat{\bar{x}}_{k}\right|\right]
\end{aligned}
$$

The $i^{\text {th }}$ component of this difference is given by:

$$
\begin{aligned}
\underline{e}_{k+1, i}= & \sum_{j=1}^{n}\left(a_{i, j}+\delta_{k, i, j}\right) x_{k, j}-\ell_{1, i, j}\left(x_{k, j}-\underline{\hat{x}}_{k, j}\right) \\
& -\frac{1}{2} \sum_{j=1}^{n}\left(\left(a_{i, j}+\left|a_{i, j}\right|\right) \underline{\hat{x}}_{k, j}+\left(a_{i, j}-\left|a_{i, j}\right|\right) \hat{\bar{x}}_{k, j}\right) \\
& -\frac{1}{2} \sum_{j=1}^{n}\left(\delta_{i, j}\left(\left|\underline{x}_{k, j}\right|+\left|\bar{x}_{k, j}\right|\right)+\operatorname{sgn}\left(a_{i, j}\right) \delta_{i, j}\left(\left|\underline{\hat{x}}_{k, j}\right|-\left|\bar{x}_{k, j}\right|\right)\right)
\end{aligned}
$$

where $\ell_{1, i, j}$ are the coefficients of the $L_{1}$ matrix. In the previous sum, let us analyse the $j^{\text {th }}$ term whether $a_{i, j}$, is positive or negative: 
- If $a_{i, j}>0$

$$
\begin{aligned}
\underline{e}_{k+1, i, j} & =\left(a_{i, j}+\delta_{k, i, j}\right) x_{k, j}-a_{i, j} \underline{\hat{x}}_{k, j}+\delta_{i, j}\left|\underline{\hat{x}}_{k, j}\right|-\ell_{1, i, j}\left(x_{k, j}-\underline{\hat{x}}_{k, j}\right) \\
& =\left(a_{i, j}+\delta_{k, i, j}-\ell_{1, i, j}\right) x_{k, j}-a_{i, j} \underline{\hat{x}}_{k, j}+\delta_{i, j}\left|\underline{\hat{x}}_{k, j}\right|+\ell_{1, i, j} \underline{\hat{x}}_{k, j} \\
\geq & \left(a_{i, j}+\delta_{k, i, j}-\ell_{1, i, j}\right) \underline{\hat{x}}_{k, j}-a_{i, j} \underline{\hat{x}}_{k, j}+\delta_{i, j}\left|\underline{\hat{x}}_{k, j}\right|+\ell_{1, i, j} \underline{\hat{x}}_{k, j} \\
& \geq \delta_{k, i, j} \underline{\hat{x}}_{k, j}+\delta_{i, j}\left|\underline{\hat{x}}_{k, j}\right| \\
& \geq 0
\end{aligned}
$$

- If $a_{i, j}<0$

$$
\begin{aligned}
\underline{e}_{k+1, i, j} & =\left(a_{i, j}+\delta_{k, i, j}\right) x_{k, j}-a_{i, j} \hat{\bar{x}}_{k, j} \delta_{i, j}\left|\hat{\bar{x}}_{k, j}\right|-\ell_{1, i, j}\left(x_{k, j}-\hat{x}_{k, j}\right) \\
& =\left(a_{i, j}+\delta_{k, i, j}-\ell_{1, i, j}\right) x_{k, j}-a_{i, j} \hat{\bar{x}}_{k, j}+\delta_{i, j}\left|\overline{\bar{x}}_{k, j}\right|+\ell_{1, i, j} \underline{\hat{x}}_{k, j} \\
& \geq\left(a_{i, j}+\delta_{k, i, j}-\ell_{1, i, j}\right) \hat{\bar{x}}_{k, j}-a_{i, j} \hat{\bar{x}}_{k, j}+\delta_{i, j}\left|\hat{\bar{x}}_{k, j}\right|+\ell_{1, i, j} \underline{\hat{x}}_{k, j} \\
& \geq \delta_{k, i, j} \hat{\bar{x}}_{k, j}+\delta_{i, j}\left|\bar{x}_{k, j}\right| \\
& \geq 0
\end{aligned}
$$

This shows that $\underline{e}_{k+1, i}$ is a sum of non negative terms and then $x_{k+1, i} \geq$ $\underline{\hat{x}}_{k+1, i}$, i.e. $x_{k+1} \geq \underline{\hat{x}}_{k+1}$. A similar calculus leads to the result $x_{k} \leq \hat{\bar{x}}_{k}$. Therefore, the property $\mathcal{H}_{k+1}$ is demonstrated and the induction principle ensures that for all $k>0$, we have $\underline{\hat{x}}_{k} \leq x_{k} \leq \hat{\bar{x}}_{k}$ which achieves the proof.

In the following, the state observer equations are denoted:

$$
\begin{aligned}
& \hat{x}_{k+1}=H_{11} \underline{\hat{x}}_{k}+H_{12} \hat{\bar{x}}_{k}+H_{13}\left|\hat{x}_{k}\right|+H_{14}\left|\hat{\bar{x}}_{k}\right|+L_{1}\left(y_{k}-C \hat{\underline{x}}_{k}\right)+B u_{k} \\
& \hat{\bar{x}}_{k+1}=H_{21} \underline{\hat{x}}_{k}+H_{22} \hat{\bar{x}}_{k}+H_{23}\left|\underline{\hat{x}}_{k}\right|+H_{24}\left|\hat{\bar{x}}_{k}\right|+L_{2}\left(y_{k}-C \overline{\bar{x}}_{k}\right)+B u_{k}
\end{aligned}
$$

with:

$$
\begin{array}{lll}
H_{11}=\frac{1}{2}(A+|A|), & H_{12}=\frac{1}{2}(A-|A|), & H_{13}=\frac{-1}{2}\left(\Delta+\Delta * S_{A}\right), \\
H_{14}=\frac{1}{2}\left(-\Delta+\Delta * S_{A}\right) & H_{21}=\frac{1}{2}(A-|A|), & H_{22}=\frac{1}{2}(A+|A|), \\
H_{23}=\frac{1}{2}\left(\Delta-\Delta * S_{A}\right), & H_{24}=\frac{1}{2}\left(\Delta+\Delta * S_{A}\right) &
\end{array}
$$

To characterize the observer's performance, let rewrite the deviations (31) as:

$$
\begin{aligned}
\underline{e}_{k+1} & =\left(A+\Delta_{k}\right) x_{k}-H_{11} \underline{\hat{x}}_{k}-H_{12} \hat{\bar{x}}_{k}-H_{13}\left|\underline{\hat{x}}_{k}\right|-H_{14}\left|\hat{\bar{x}}_{k}\right|-L_{1}\left(y_{k}-C \underline{\hat{x}}_{k}\right) \\
& =\left(H_{11}-L_{1} C\right) \underline{e}_{k}-H_{12} \bar{e}_{k}-\Delta_{k} x_{k}-H_{13}\left|\underline{x}_{k}\right|-H_{14}\left|\hat{\bar{x}}_{k}\right| \\
\bar{e}_{k+1} & =H_{21} \underline{\hat{x}}_{k}+H_{22} \overline{\bar{x}}_{k}-\left(A+\Delta_{k}\right) x_{k}+H_{23}\left|\underline{\hat{x}}_{k}\right|+H_{23}\left|\hat{\bar{x}}_{k}\right|+L_{2}\left(y_{k}-C \hat{\bar{x}}_{k}\right) \\
& =-H_{21} \underline{e}_{k}+\left(H_{22}-L_{2} C\right) \bar{e}_{k}-\Delta_{k} x_{k}-H_{23}\left|\underline{\hat{x}}_{k}\right|-H_{24}\left|\hat{\bar{x}}_{k}\right|
\end{aligned}
$$

The two deviations defined in (37) are gathered in the following augmented state estimation error: $e_{k}^{T}=\left[\begin{array}{ll}\underline{e}_{k}^{T} & \bar{e}_{k}^{T}\end{array}\right]$ and ruled by the following discrete time system:

$$
e_{k+1}=\left(A_{0}-L C_{0}\right) e_{k}+B_{0} \omega_{k}
$$


where the matrices $A_{0}, L, C_{0}$ and $B_{0}$ and the input signal $\omega_{k}$ are defined by

$$
\begin{aligned}
& A_{0}=\left[\begin{array}{cc}
H_{11} & -H_{12} \\
-H_{21} & H_{22}
\end{array}\right], \quad B_{0}=\left[\begin{array}{ccc}
I_{n} & -H_{13} & -H_{14} \\
-I_{n} & -H_{23} & -H_{24}
\end{array}\right], \quad L=\left[\begin{array}{cc}
L_{1} & 0 \\
0 & L_{2}
\end{array}\right], \\
& C_{0}=\left[\begin{array}{cc}
C & 0 \\
0 & C
\end{array}\right] \quad \omega_{k}=\left[\begin{array}{lll}
\left(\Delta_{k} x_{k}\right)^{T} & \left|\underline{\hat{x}}_{k}\right|^{T} & \left|\hat{\bar{x}}_{k}\right|^{T}
\end{array}\right]^{T}
\end{aligned}
$$

It is then necessary to analyze the dynamics of the deviations in (38) to adjust the gains $L_{1}$ and $L_{2}$ in $L$ to ensure the boundedness and the positivity of $\underline{e}_{k}$ and $\bar{e}_{k}$. This can be done as the result of an LMI optimisation procedure, as stated in the following theorem.

Theorem 3. Given a scalar $\gamma>0$, the system (29) is an observer of system (1), if there exists a matrix $P=P^{T}>0 \in \mathbb{R}^{2 n \times 2 n}$, matrices $K_{1}$ and $K_{2} \in \mathbb{R}^{n \times p}$ and invertible matrices $G_{1}$ and $G_{2} \in \mathbb{R}^{n \times n}$, such that the following LMI holds

$$
\left[\begin{array}{ccc}
I_{2 n}-P & 0 & A_{0}^{T} G-C_{0}^{T} K^{T} \\
0 & -\gamma^{2} I_{m} & B_{0}^{T} G \\
G^{T} A_{0}-K C_{0} & G^{T} B_{0} & P-G^{T}-G
\end{array}\right]<0
$$

where $G_{0}$ and $K_{0}$ are the block diagonal matrices defined by:

$$
G=\left[\begin{array}{cc}
G_{1} & 0 \\
0 & G_{2}
\end{array}\right] \quad K=\left[\begin{array}{cc}
K_{1} & 0 \\
0 & K_{2}
\end{array}\right]
$$

After solving the LMI (40), the observer gain is defined by:

$$
L=\left(G^{T}\right)^{-1} K
$$

Proof. The evolution of the deviations is governed by (38), depending on the gains $L_{1}$ and $L_{2}$. The objective of the synthesis of the parameters $L_{1}$ and $L_{2}$ is twofold: ensure the stability of the matrix $A_{0}-L C_{0}$ and reduce the influence of the disturbance-like term $\omega$ on the error $e$. This is translated as follows:

$$
\begin{aligned}
& \lim _{k \rightarrow \infty} e_{k}=0 \quad \text { if } \quad \omega_{k}=0 \\
& \frac{\left\|e_{k}\right\|_{2}}{\left\|\omega_{k}\right\|_{2}}<\gamma \quad \text { if } \quad \omega_{k} \neq 0
\end{aligned}
$$

Let $V\left(e_{k}\right)=e_{k}^{T} P e_{k}$ be a Lyapunov candidate function. The state estimation error convergence is guaranteed and the gain $\mathcal{L}_{2}$ of the transfer from $\omega_{k}$ to $e_{k}$ is bounded by $\gamma$ if:

$$
V\left(e_{k+1}\right)-V\left(e_{k}\right)+e_{k}^{T} e_{k}-\gamma^{2} \omega_{k}^{T} \omega_{k}<0
$$

Defining $\Psi=A_{0}-L C_{0}$ and using (38), one obtains:

$$
V\left(e_{k+1}\right)-V\left(e_{k}\right)=e_{k}^{T} \Psi^{T} P \Psi e_{k}+2 e_{k}^{T} \Psi^{T} P B_{0} \omega_{k}+\omega_{k}^{T} B_{0}^{T} P B_{0} \omega_{k}-e_{k}^{T} P e_{x}
$$

and, with (45), (44) becomes:

$$
e_{k}^{T}\left(\Psi^{T} P \Psi-P+I\right) e_{k}+2 e_{k}^{T} \Psi^{T} P B_{0} \omega_{k}+\omega_{k}^{T} B_{0}^{T} P B_{0} \omega_{k}-\gamma^{2} \omega_{k}^{T} \omega_{k}<0
$$


The constraint (46) is ensured if there exists matrices $P$ and $L$ satisfying:

$$
\left[\begin{array}{cc}
I-P & 0 \\
0 & -\gamma^{2} I_{m}
\end{array}\right]+\left[\begin{array}{l}
\Psi^{T} \\
B_{0}^{T}
\end{array}\right] P\left[\begin{array}{ll}
\Psi & B_{0}
\end{array}\right]<0
$$

From lemma 3 of [13], searching $P$ and $L$ satisfying (47) is known to be equivalent to searching $P, G$ and $L$ satisfying

$$
\left[\begin{array}{ccc}
I-P & 0 & \Psi^{T} G \\
0 & -\gamma^{2} I_{m} & B_{0}^{T} G \\
G^{T} \Psi & G^{T} B_{0} & P-G-G^{T}
\end{array}\right]<0
$$

which is the LMI (40), up to the variable change $G^{T} L=K$.

The Theorem 3 provides an observer design fulfilling the constraints (43). However, this approach suffers from a certain conservatism mainly due to the structure of the equations (36) expressing the evolution of reconstruction errors. Indeed, in (36), and thus in (38), the dependence between the variables $x_{k}$ and $\left|x_{k}\right|$ is not taken into account. Accordingly to (21), the magnitude of the lower and upper bounds of the state estimate can be expressed as a multi-model based on

$$
\begin{aligned}
& \left|\underline{\hat{x}}_{k}\right|=\sum_{i=1}^{r} \mu_{i}^{1}\left(\underline{\hat{x}}_{k}\right) E_{i} \underline{\hat{x}}_{k} \\
& \left|\hat{\bar{x}}_{k}\right|=\sum_{j=1}^{r} \mu_{j}^{2}\left(\hat{\bar{x}}_{k}\right) E_{j} \hat{\bar{x}}_{k}
\end{aligned}
$$

where the activating functions $\mu_{i}^{1}$ and $\mu_{j}^{2}$ satisfy the convex sum property (23) and $r=2^{n}$. Using this multi-model expression of the magnitude of the lower and upper bounds of the state estimation error (37), these equations become

$$
\begin{aligned}
& \underline{e}_{k+1}=\left(H_{11}-L_{1} C\right) \underline{e}_{k}-H_{12} \bar{e}_{k}-\Delta x_{k}-H_{13} \sum_{i=1}^{r} \mu_{i}^{1}(\underline{\hat{x}}) E_{i} \underline{\hat{x}}-H_{14} \sum_{j=1}^{r} \mu_{j}^{2}(\hat{\bar{x}}) E_{j} \hat{\bar{x}} \\
& \bar{e}_{k+1}=-H_{21} \underline{e}_{k}+\left(H_{22}-L_{2} C\right) \bar{e}_{k}-\Delta_{k} x_{k}-H_{23} \sum_{i=1}^{r} \mu_{i}^{1}(\underline{\hat{x}}) E_{i} \underline{\hat{x}}-H_{24} \sum_{j=1}^{r} \mu_{j}^{2}(\hat{\bar{x}}) E_{i} \hat{\bar{x}}
\end{aligned}
$$

Since the functions $\mu_{i}^{1}$ and $\mu_{j}^{2}$ satisfy (23), these two equations can be written as

$$
\begin{aligned}
\underline{e}_{k+1}= & \sum_{i=1}^{r} \sum_{j=1}^{r} \mu_{i}^{1}(\underline{\hat{x}}) \mu_{j}^{2}(\hat{\bar{x}})\left(\left(H_{11}-L_{1} C+H_{13} E_{i}\right) \underline{e}_{k}\right. \\
& \left.-\left(H_{12}+H_{14} E_{j}\right) \bar{e}_{k}-\left(\Delta+H_{13} E_{i}+H_{14} E_{j}\right) x_{k}\right) \\
\bar{e}_{k+1}= & \sum_{i=1}^{r} \sum_{j=1}^{r} \mu_{i}^{1}(\underline{\hat{x}}) \mu_{j}^{2}(\hat{\bar{x}})\left(-\left(H_{21}-H_{23} E_{i}\right) \underline{e}_{k}\right. \\
& \left.+\left(H_{22}-L_{2} C-H_{24} E_{j}\right) \bar{e}_{k}-\left(\Delta+H_{23} E_{i}+H_{24} E_{j}\right) x_{k}\right)
\end{aligned}
$$

With (51), the augmented system (38) becomes

$$
e_{k+1}=\sum_{i=1}^{r} \sum_{j=1}^{r} \mu_{i}^{1}(\underline{\hat{x}}) \mu_{j}^{2}(\hat{\bar{x}})\left(\left(A_{i, j}-L C_{0}\right) e_{k}+B_{i, j} x_{k}\right)
$$


where $L$ and $C_{0}$ are already defined in (39) and $A_{i j}$ and $B_{i j}$ are given by

$$
\begin{aligned}
A_{i, j} & =\left[\begin{array}{cc}
H_{11}+H_{13} E_{i} & -\left(H_{12}+H_{14} E_{j}\right) \\
-\left(H_{21}-H_{23} E_{i}\right) & H_{22}-H_{24} E_{j}
\end{array}\right] \\
B_{i, j} & =-\left[\begin{array}{l}
\Delta+H_{13} E_{i}+H_{14} E_{j} \\
\Delta+H_{23} E_{i}+H_{24} E_{j}
\end{array}\right]
\end{aligned}
$$

This leads to the following theorem.

Theorem 4. Given a scalar $\gamma>0$, the system (29) is an observer of system (1), if there exists a matrix $P=P^{T}>0 \in \mathbb{R}^{2 n \times 2 n}$, matrices $K_{1}$ and $K_{2} \in \mathbb{R}^{n \times p}$ and invertible matrices $G_{1}$ and $G_{2} \in \mathbb{R}^{n \times n}$, such that

$$
\mathcal{M}_{i j}+\mathcal{M}_{j i}<0, i, j=1, \ldots, r
$$

where $\mathcal{M}_{i j}$ is defined by

$$
\mathcal{M}_{i j}=\left[\begin{array}{ccc}
I_{2 n}-P & 0 & A_{i j}^{T} G-C_{0}^{T} K^{T} \\
0 & -\gamma^{2} I_{n} & B_{i j}^{T} G \\
G^{T} A_{i j}-K C_{0} & G^{T} B_{i j} & P-G^{T}-G
\end{array}\right]
$$

and where $G_{0}$ and $K_{0}$ are the block diagonal matrices defined in (41). After solving the LMI (54), the observer gain is defined by (42).

Proof. The proof follows the same lines of the one of Theorem 3: firstly the quadratic Lyapunov function $V\left(e_{k}\right)=e_{k}^{T} P e_{k}$ is defined and then its decreasing along the trajectory of $(52)$ is studied, taking benefits of the positiveness of the activating functions satisfying (23).

Remark 2. In Theorems 3 and 4 , the attenuation level $\gamma$ can be minimized, by considering the classical variable change $\bar{\gamma}=\gamma^{2}$ and by minimizing $\bar{\gamma}$ under the LMI constraints of Theorems 3 or 4.

Remark 3. The corollary 1 can be used to introduce some additional degrees of freedom in the LMI optimization and thus reduce the conservatism implied by a simple quadratic Lyapunov function. Nevertheless a trade-off should thus be made between relaxing the conservatism and limiting the computational burden of seeking numerous LMI variables.

\subsection{Example}

The example 2.4 is continued with the same system matrices (17) and an output defined by $C=\left[\begin{array}{ll}1 & 0\end{array}\right]$. Figure 2 depicts the evolution of the two inputs and the two states. As in example 2.4, the red plot is a possible state trajectory given the parameter uncertainty and the green and blue ones are the upper and lower bounds of each state trajectory. Solving the LMI conditions (54), the 
following solutions are obtained

$$
\begin{gathered}
P=\left(\begin{array}{cccc}
3.011 & 0.328 & 1.724 & 0.636 \\
0.328 & 12.266 & 0.636 & 0.727 \\
1.724 & 0.636 & 3.011 & 0.328 \\
0.636 & 0.727 & 0.328 & 12.266
\end{array}\right), \\
G_{1}=\left(\begin{array}{cc}
2.766 & .424 \\
0.424 & 18.359
\end{array}\right), \quad G_{2}=\left(\begin{array}{cc}
2.766 & 0.424 \\
0.424 & 18.359
\end{array}\right) \\
K_{1}=\left(\begin{array}{ll}
2.522 & -3.168
\end{array}\right)^{T}, \quad K_{2}=\left(\begin{array}{ll}
2.522 & -3.168
\end{array}\right)^{T}
\end{gathered}
$$

resulting in the observer gains (42)

$$
L_{1}=\left[\begin{array}{ll}
0.942 & -0.194
\end{array}\right]^{T}, \quad L_{2}=\left[\begin{array}{ll}
0.942 & -0.194
\end{array}\right]^{T}
$$

With these gains, the observer can be simulated with (29) and one can see, on the figure 3, that the actual system state (red plot) appears to be in the envelope provided by the interval observer (green and red lines). It can also be pointed out that the envelope provided by the interval observer gives a thinner estimate of the actual state of the system. The comparison of figures 2 and 3 clearly shows the interest of the interval observer: firstly it reconstructs the interval system state from the measured inputs and outputs, secondly the obtained radius of the envelope is smaller than the one provided by the direct simulation of the state equation interval.
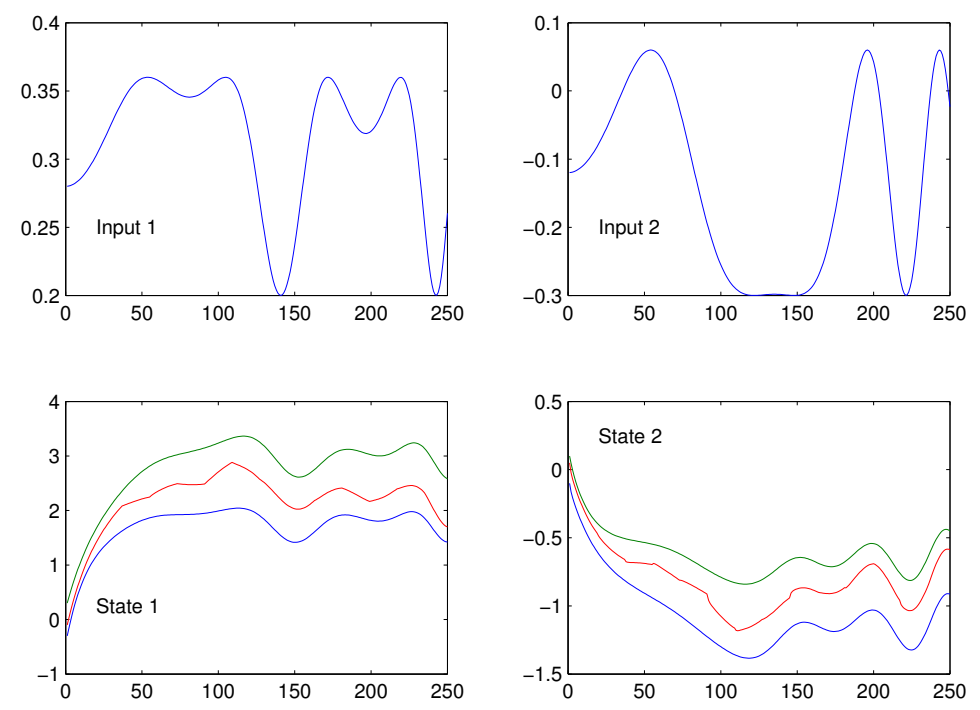

Figure 2: Simulation of a system with interval parameters

Remark 4. Following [5], additional degrees of freedom can be introduced in the observer design by tuning an extra parameter, denoted $F$, in the following 

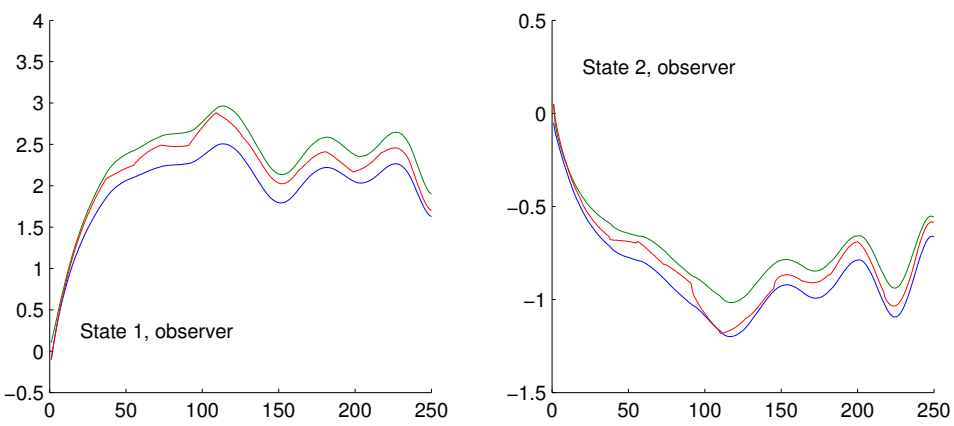

Figure 3: State reconstruction of a system with interval parameters

observer definition:

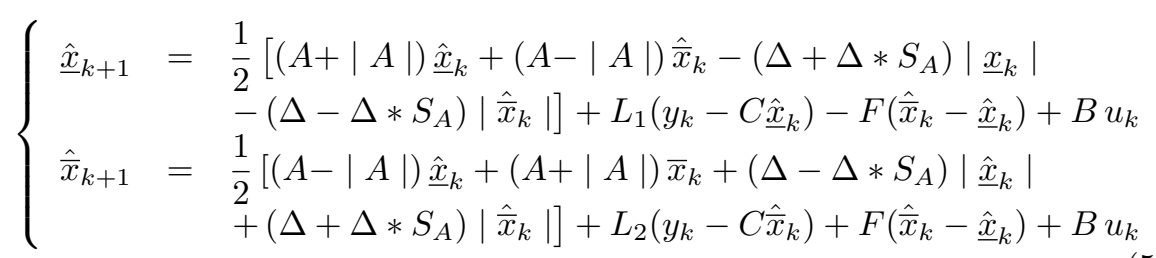

\section{Conclusion}

The proposed approach provides a systematic state estimation for a system with uncertain but bounded parameters. It significantly differs from earlier work in showing that the system model can be used to simultaneously model the lower and upper bounds of the system state trajectory. Both models are structured in a polytopic form and allow to synthesize an interval observer. This approach can easily be extended to other situations. On the one hand, the inclusion of uncertainty affecting all system state matrices appears as a direct extension. On the other hand, one can consider changing the observer interval to encompass the case of interval measures of the inputs and outputs.

\section{Appendix A. Some details on the multi-model form of the absolute value function}

The simulation of the interval model (18) does not pose any particular difficulty, except the fact that the equation number is twice the one of the initial system. However, the presence of the absolute value operator can make tedious the stability analysis and the observer design. For this reason, it is proposed to reformulate the interval model by replacing the absolute value operator by a polytopic model that is more easily handled. 
To begin with, let us consider a two component vector $z=\left[\begin{array}{ll}z_{1} & z_{2}\end{array}\right]^{T}$. Its component wise absolute value is then defined by $|z|=\left[\left|z_{1}\right| \quad\left|z_{2}\right|\right]^{T}$ and it can be expressed by:

$$
\left\{\begin{array}{l}
\left|z_{i}\right|=\mu_{i 1}\left(z_{i}\right) \cdot\left(z_{i}\right)+\mu_{i 2}\left(z_{i}\right) \cdot\left(-z_{i}\right) \\
\mu_{i 1}\left(z_{i}\right)=\frac{1+\operatorname{sgn}\left(z_{i}\right)}{2} \\
\mu_{i 2}\left(z_{i}\right)=1-\mu_{i 1}\left(z_{i}\right)
\end{array}, i=1,2\right.
$$

Each scalar $\left|z_{i}\right|$ is expressed in terms of both its own weight functions $\mu_{i 1}$ and $\mu_{i 2}$. In order to express all of these scalars with the same weight function set, they can be equivalently written as:

$$
\left|z_{i}\right|=\left(\mu_{i 1}+\mu_{i 2}\right)\left(\mu_{i 1} \cdot\left(z_{i}\right)+\mu_{i 2} \cdot\left(-z_{i}\right)\right), i=1,2
$$

Defining $\mu_{1}=\mu_{11} \mu_{21}, \mu_{2}=\mu_{11} \mu_{22}, \mu_{3}=\mu_{12} \mu_{21}, \mu_{4}=\mu_{12} \mu_{22}$ and

$$
E_{1}=\left[\begin{array}{ll}
1 & 0 \\
0 & 1
\end{array}\right] \quad E_{2}=\left[\begin{array}{cc}
1 & 0 \\
0 & -1
\end{array}\right] \quad E_{3}=\left[\begin{array}{cc}
-1 & 0 \\
0 & 1
\end{array}\right] \quad E_{4}=\left[\begin{array}{cc}
-1 & 0 \\
0 & -1
\end{array}\right]
$$

the absolute values $\left|z_{1}\right|$ and $\left|z_{2}\right|$ are expressed under a polytopic form which depends on the same weight function set, as follows :

$$
|z|=\sum_{i=1}^{4} \mu_{i} E_{i} z
$$

Based on the previous developments in the two dimensional case, the polytopic writing can easily be generalized to any vector $z \in \mathbb{R}^{r}$, as described in [37] or [29]. The common weighting functions $\mu_{i}(z)$ are defined by products of the weighting functions depending on each vector component (namely $\mu_{i 1}\left(z_{i}\right)$ and $\left.\mu_{i 2}\left(z_{i}\right)\right)$ and the matrices $E_{i}$ are diagonal matrices which entries are equal to the extremal values of $\mu_{i 1}\left(z_{i}\right)$ and $\mu_{i 2}\left(z_{i}\right)$, i.e. 1 or -1 .

[1] Ahn HS, Moore KL, Chen YQ. Stability analysis of discrete-time iterative learning control systems with interval uncertainty. Automatica 2007; 43: 892-902.

[2] Alcaraz-Gonzalez V, Genovesi A, Harmand J, Gonzalez AV, Rapaport A, Steyer JP. Robust exponential nonlinear interval observers for a class of lumped models useful in chemical and biochemical engineering - Application to a wastewater treatment plant. Proc. International Workshop on Application of Interval Analysis to Systems and Control, Girona, Spain, 1999.

[3] Ben Chabane S, Stoica Maniu C, Alamo T, Camacho EF, Dumur D. Ellipsoidal state estimation for systems with interval uncertainties. Proc. 53rd IEEE Conference on Decision and Control, Los Angeles, California, USA, 2014. 
[4] Bernard O, Gouz JL. Closed loop observers bundle for uncertain biotechnological models. Journal of Process Control 2004; 14: 765-774.

[5] Bolajraf M, Rami M, Tadeo F. Robust interval observer with uncertainties in the output. Proc. 18th Mediterranean Conference on Control Automation, Marrakech, Morocco, 2010.

[6] Chambon E, Burlion L, Apkarian P. Overview of linear time-invariant interval observer design : towards a non-smooth optimisation-based approach. IET Control Theory and Applications 2016;10 (11): 1258-1268.

[7] Chebotarev S, Efimov D, Raissi T, Zolghadri A. Interval state observer for continuous-time LPV systems with $L_{1} / L_{2}$ performance. Automatica 2015; 49 (1): 200-205.

[8] Chen SH, Lian HD, Yang XW. Interval eigenvalue analysis for structures with interval parameters. Finite Elements in Analysis and Design 2003; 39: 419-43.

[9] Chen X, Lam J. Positive state-bounding observer for interval positive systems under $L 1$ performance, Proc. 33rd Chinese Control Conference, Nanjing, China, 2014.

[10] Cherrier E, Boutayeb B, Ragot J. Evaluation des bornes d'un systme incertain ; approche intervalle. Journal Europen des Systmes Automatiss, Numro spcial "Applications des outils de calculs ensemblistes" 2004; 37(9): 1181-1192 (in French).

[11] Chisci L, Garulli A, Zappa G. Recursive state bounding by parallelotopes. Automatica 1996; 32 (7): 1049-1055.

[12] Combastel C. A state bounding observer for uncertain non-linear continuous-time systems based on zonotopes. Proc. 44th IEEE Conference on Decision and Control and the European Control Conference, Seville, Spain, 2005.

[13] Delmotte F, Guerra TM, Ksantini M. Continuous Takagi-Sugeno's models: Reduction of the number of LMI conditions in various fuzzy control design technics. IEEE Transactions on Fuzzy Systems 2007; 15(3): 426-438.

[14] Dinh T, Mazenc F, Niculescu SI. Interval observer composed of observers for nonlinear systems. Proc. 13th European Control Conference, Strasbourg, France, 2014.

[15] Efimov D, Perruquetti W, Raissi T, Zolghadri A. On interval observer design for time-invariant discrete-time systems. Proc. 12th European Control Conference, Zurich, Switzerland, 2013.

[16] Efimov D, Raissi T, Chebotarev S, Zolghadri A. Interval state observer for nonlinear time varying systems. Automatica 2013; 49 (1): 200-205. 
[17] Ellero N, Gucik-Derigny D, Henry D. Unknown input interval observer for uncertain linear time invariant systems. Proc. 11th International Conference on Control, Belfast, UK, 2016.

[18] Faydasicoka O, Arikb S. A new upper bound for the norm of interval matrices with application to robust stability analysis of delayed neural networks. Neural Networks 2013; 44: 64-71.

[19] Goffaux G, Wouwer AV, Bernard O. Improving continuous-discrete interval observers with application to microalgae-based bioprocesses. Journal of Process Control 2009; 19: 1182-1190.

[20] Goffaux G, Remy M, Wouwer AV. Continuous-discrete confidence interval observer - Application to vehicle positioning. Information Fusion 2013; 14: 541-550.

[21] Gouz J-L, Rapaport A, Hadj-Sadok MZ. Interval observers for uncertain biological systems. Ecological modelling 2000; 133(1-2): 45-56.

[22] Gucik-Derigny D, Rassi T, Zolghadri A. Interval state and unknown inputs estimation for linear time-invariant systems. Proc. 19th IFAC World Congress, Cape Town, South Africa, 2014.

[23] Haoa C, Lianga Y, Roy A. Equivalency between vertices and centerscoupled-with-radii principal component analyses for interval data. Statistics and Probability Letters 2015; 106: 113-120.

[24] Jaulin L. Nonlinear bounded-error state estimation of continuous time systems. Automatica 2002; 38 (2): 1079-1082.

[25] Lamouchi R, Amairi M, Rassi T, Aoun M. Interval observer design for linear parameter-varying systems subject to component faults. Proc. 24th Mediterranean Conference on Control and Automation, Athens, Greece, 2016.

[26] Langowski R, Brdys MA. Monitoring of chlorine concentration in drinking water distribution systems using an interval estimator. International Journal Applied Math. Computer Science 2007;17 (2): 199-216.

[27] Martnez-Sibaja A, Astorga-Zaragoza CM, Alvarado-Lassman A, PosadaGmez R, Aguila-Rodrguez G, Rodrguez-Jarquin JP, Adam-Medina M. Simplified interval observer scheme: A new approach for fault diagnosis in instruments. Sensors 2011; 11: 612-622.

[28] Moisan M., Bernard O., Gouz J-L. Near optimal interval observers bundle for uncertain bioreactors. Automatica 2009; 45: 291-295.

[29] Nagy AM, Mourot G, Marx B, Ragot J, Schutz G. Systematic multimodeling methodology applied to an activated sludge reactor model. Industrial \&3 Engineering Chemistry Research 2010; 46(6): 2790-2799. 
[30] Pastravanu O, Matcovschi MH. Diagonal stability of interval matrices and applications. Linear Algebra and its Applications 2010; 433: 1646-1658.

[31] Polyakov A, Efimov D, Perruquetti W, Richard J-P. Interval observer approach to output stabilization of time-varying input delay systems. Proc. 12th European Control Conference, Zurich, Switzerland, 2013.

[32] Puri A and Varaiya P. E-Approximation of differential inclusions. Proc. 34th Conference on Decision and Control, New Orleans, LA, USA, 1995.

[33] Rassi T. Mthodes ensemblistes pour l'estimation d'tat et de paramtres. PhD Thesis of the Universit Paris XII Val de Marne, 2004 (in French).

[34] Rotondo D, Fernadez-Canti RM, Tornil-Sin S, Blesa J, Puig V. Robust fault diagnosis of proton exchange membrane fuel cells using a Takagi-Sugeno interval observer approach. International Journal of Hydrogen Energy 2016; 41: $1875-1886$.

[35] Shao G, Su J. Sensitivity and inverse analysis methods for parameter intervals. Journal of Rock Mechanics and Geotechnical Engineering 2010; 2 (3): $274-280$.

[36] Shu Z, Lam J, Gao H, Du B, Wu L. Positive observers and dynamic outputfeedback controllers for interval positive linear systems. IEEE Transactions on Automatic Control 2008; 55 (10): 3209-3222

[37] Tanaka K, Wang HO. Fuzzy Control Systems Design and Analysis. A Linear Matrix Inequality Approach. John Wiley \& Sons; 2001.

[38] Utkin LV, Chekh AI. A new robust model of one-class classification by interval-valued training data using the triangular kernel. Neural Networks 2015; 69: 99-110.

[39] Wang C, Li J, Guo P. The normalized interval regression model with outlier detection and its real-world application to house pricing problems. Fuzzy Sets and Systems 2015; 274: 109-123.

[40] Wang Y, Bevly D, Rajamani R. Interval observer design for LPV systems with parametric uncertainty. Automatica 2015; 60 (10): 79-85. 